\title{
Dosimetric effects of the Smit sleeve on high-dose- rate brachytherapy tandem and ovoids plans for patients with locally advanced cervical cancer
}

\author{
Shahil Mehta, MD!, Benjamin Farnia, MD!, Alberto de la Zerda, PhD², Robabeh Rahimi, PhD!, Aaron Wolfson, MD! \\ Lorraine Portelance, MDl \\ 'Department of Radiation Oncology, Sylvester Comprehensive Cancer Center/University of Miami Miller School of Medicine, Miami, FL, \\ United States, ${ }^{2}$ Department of Radiation Oncology. Jackson Memorial Hospital/University of Miami Miller School of Medicine, Miami, FL, \\ United States
}

\begin{abstract}
Purpose: Smit sleeves are used to facilitate insertion of the intrauterine tandem during brachytherapy for cervical cancer. When a tandem and ovoids system is used the base of the Smit sleeve displaces the ovoids distally. The dosimetric impact of this displacement is not known. Herein we performed a dosimetric analysis to quantify this impact on the integral dose and dose delivered to the organs at risk (OARs).

Material and methods: Eleven high-dose-rate brachytherapy plans in which a Smit sleeve was used with a tandem and ovoids were reviewed. A second set of plans was generated modifying the position of the ovoids to simulate absence of the Smit sleeve. The high-risk clinical tumor volume (HR-CTV) dose coverage was maintained the same for both sets of plans by appropriately rescaling the dwell times of the simulated plan. The mean integral dose, $\mathrm{D}_{2 c \mathrm{c}}$ to the OARs (bladder, bowel, sigmoid and rectum) and the ICRU rectum point dose were compared between the original and modified plans using a paired two-sample $t$-test.

Results: Simulating removal of the Smit sleeve was associated with an average reduction in the mean integral dose of $6.1 \%(p<0.001)$ and an average reduction of $10.9 \%(p=0.004)$ to the rectal $\mathrm{D}_{2 \mathrm{cc}}$. Doses to the remaining OARs decreased to a lesser magnitude with only that of the sigmoid being statistically significant.

Conclusions: The use of a Smit sleeve with a tandem and ovoids system could lead to the delivery of a higher mean integral dose to achieve similar HR-CTV coverage. In addition, it could increase the dose to surrounding OARs, primarily the rectum. The clinical significance of these findings is unknown, but the potential dosimetric impact of using a Smit sleeve should be taken into consideration during the planning when this device is used.

J Contemp Brachytherapy 2019; 11, 6: 584-588 DOI: https://doi.org/10.5114/jcb.2019.90435
\end{abstract}

Key words: brachytherapy, Smit sleeve, cervical cancer, dosimetry, integral dose, rectal dose.

\section{Purpose}

The Smit sleeve is a device developed by Elekta (Veenendaal, The Netherlands). It is a disposable, silicone-based indwelling intrauterine tube that can be used during brachytherapy treatment for cervical cancer to allow for easier insertion of the tandem [1]. The tube has a flange on the distal side, which acts as a stopper during tandem insertion $[1,2,3,4]$. The cannula of the Smit sleeve marginally increases the radius around the tandem, while the base of the Smit sleeve, which lies distal to the external cervical os, displaces the ovoids distally. In some tandem and ovoids systems, the flange on the tandem also lies between the cervix and the superior surface of the ovoids and must be accounted for when measuring the total displacement of the ovoids. In the tandem and ring systems, the base of the Smit sleeve fits in the opening of the ring and the use of this device has no impact on the geometry of the implant. The Smit sleeve on a tandem and ovoids model can be seen in Figure 1, where the combined thickness of the Smit sleeve and the flange is $5 \mathrm{~mm}$, pushing the ovoids away from the target tissue. Distally displacing the ovoids by $5 \mathrm{~mm}$ may impact the dose delivered to the high-risk clinical target volume (HR-CTV), organs at risk (OARs), vaginal mucosa, and the integral dose (ID).

Previous studies have evaluated the dosimetric effects of applicator shifts and have found that shifts of a few millimeters can lead to large deviations in dose distribution, potentially leading to a shift in dose from the 
target volume to OARs $[5,6,7,8]$. One study, which retrospectively simulated tandem and ovoid shifts, found that a displacement by $3 \mathrm{~mm}$ cranially or caudally could cause greater than a $10 \%$ dosimetric change, showing that dose errors can occur when the reconstructed applicator position and actual applicator position differ [6]. These changes in dosimetric planning caused by tandem and ovoid displacements support the idea that the presence of the Smit sleeve may impact high-dose-rate (HDR) brachytherapy dose distribution.

To our knowledge no previous studies have assessed the impact of Smit sleeve usage on dosimetric planning. This technical note summarizes the dosimetric effects of the Smit sleeve on HDR brachytherapy tandem and voids plans by comparing plans in which a Smit sleeve was used to paired simulated plans in which the ovoids were shifted proximally to simulate removal of the device. HR-CTV coverage $\left(D_{90}\right)$ was kept the same between both plans set, by adjusting the dwell times of the simulated plan. The endpoints included the maximal doses delivered to $2 \mathrm{cc}\left(\mathrm{D}_{2 \mathrm{cc}}\right)$ of the bladder, bowel, rectum, and sigmoid and to the International Commission on Radiation Units and Measurements (ICRU) rectal point, the latter of which was examined in response to sub-analysis of the EMBRACE study, which demonstrated that the ICRU rectal point is a predictive factor of grade 2 or higher vaginal toxicity, with grade $\geq 2$ toxicity increasing from $20 \%$ to $34 \%$ when this point dose increased from 65 to $85 \mathrm{~Gy}$ $\mathrm{EQD}_{2}$ [9].

\section{Material and methods}

The brachytherapy plans of 11 patients with locally advanced cervical cancer (LACC) International Federation of Gynecology and Obstetrics Stages (FIGO) IB2-IVA who received HDR brachytherapy (Elekta's microSelectron with iridium-192 [ ${ }^{192} \mathrm{Ir}$ ] source) with the use of a Smit sleeve and a tandem and ovoids system were identified through an institutional registry and selected for this do-
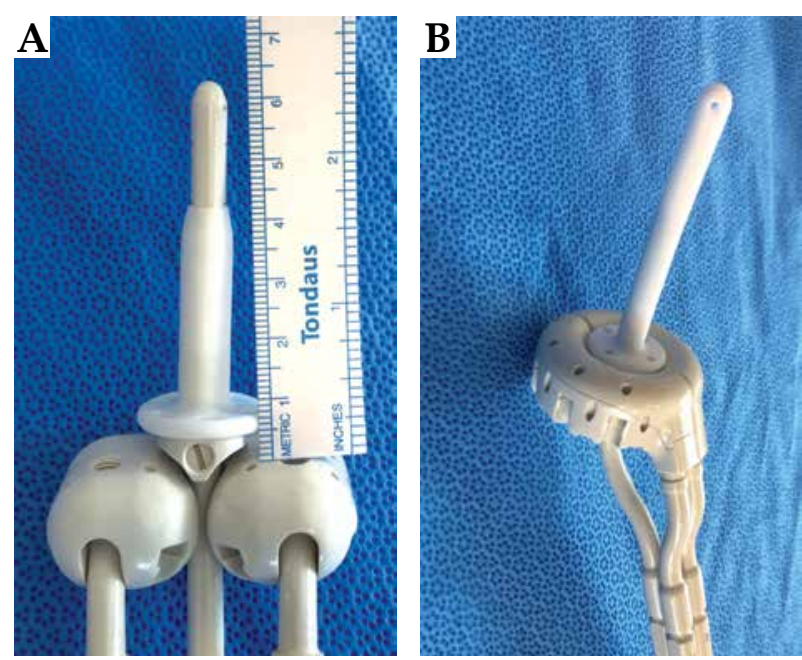

Fig. 1. Smit sleeve with a tandem and ovoids (A) compared to a tandem and ring (B). A) The distal displacement of the ovoids caused by the Smit sleeve, moving the ovoids away from the target. The use of a Smit sleeve with a tandem and ring applicators system does not have the same consequence on the applicators' geometry

simetric analysis. Brachytherapy applicators' characteristics are listed in Table 1. Different tandem and ovoid sizes were used based on patient anatomy.

All treatment plans were volume-based, built on a planning pelvic CT scan (2.5 mm slice thickness) performed with the applicators in place. The treatment planning system utilized was the Oncentra Brachy Version 4.5.3 (Elekta, Veenendaal, The Netherlands). The applicators were reconstructed and the HR-CTV, bladder, bowel, rectum, and sigmoid were contoured by the treating radiation oncologist. The prescription dose to the HR-CTV was either four fractions at $700 \mathrm{cGy}$ per fraction $(n=6)$ or three fractions at $800 \mathrm{cGy}$ per fraction $(n=5)$. The target dose coverage of the HR-CTV was at least $90 \%$ for all

Table 1. Tumor and treatment characteristics

\begin{tabular}{|c|c|c|c|c|c|c|}
\hline Fraction & Stage & $\begin{array}{c}\text { Prescribed dose } \\
\text { (Gy) }\end{array}$ & $\begin{array}{l}\text { Number of frac- } \\
\text { tions }\end{array}$ & $\begin{array}{c}\text { Ovoid diameter } \\
(\mathrm{mm})\end{array}$ & Tandem angle $\left({ }^{\circ}\right)$ & $\begin{array}{c}\text { Target HR-CTV } \\
\text { coverage (\%) }\end{array}$ \\
\hline 1 & IB2 & 7 & 4 & 30 & 30 & 95 \\
\hline 2 & IB2 & 7 & 4 & 30 & 30 & 95 \\
\hline 3 & IB2 & 8 & 3 & 20 & 30 & 95 \\
\hline 4 & IB2 & 8 & 3 & 20 & 30 & 95 \\
\hline 5 & IIIB & 8 & 3 & 25 & 30 & 90 \\
\hline 6 & IIIB & 8 & 3 & 25 & 30 & 90 \\
\hline 7 & IIIB & 8 & 3 & 30 & 30 & 95 \\
\hline 8 & $\| B$ & 7 & 4 & 20 & 30 & 90 \\
\hline 9 & $\| B$ & 7 & 4 & 25 & 30 & 90 \\
\hline 10 & $\| B$ & 7 & 4 & 25 & 30 & 90 \\
\hline 11 & $\| B$ & 7 & 4 & 25 & 30 & 90 \\
\hline
\end{tabular}




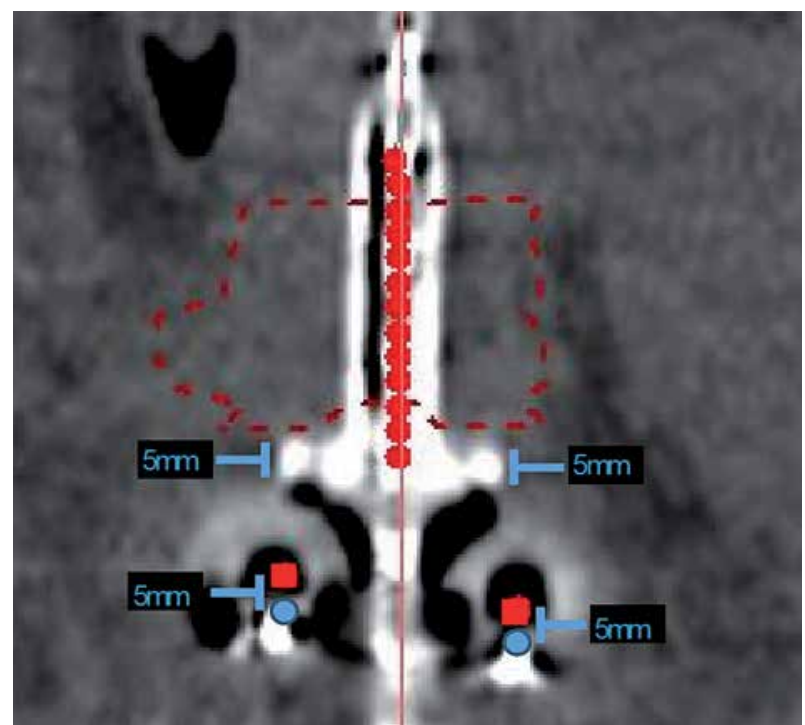

Fig. 2. Coronal view of the planning CT of the Smit sleeve and tandem and ovoids delineating the simulated $5 \mathrm{~mm}$ proximal shift parallel to the flange of the Smit sleeve. Original source positions are displayed in blue and modified source positions are displayed in red

plans and as high as $95 \%$ for some implants when the dose to the OARs allowed for increased HR-CTV coverage.

For the dosimetric analysis, the initial brachytherapy plans were modified to simulate removal of the Smit sleeve by shifting the ovoids proximally $5 \mathrm{~mm}$, along the tandem. This process is displayed in Figure 2. The goal of this shift was to simulate a plan in which the Smit sleeve was absent, allowing for the ovoids to lie adjacent to the external cervical os. After shifting the ovoids as described above, the dose distribution was re-scaled by adjusting the dwell times uniformly so as to provide the same HR-CTV dose-volume coverage as the initial plan. As a result, the HR-CTV coverage $\left(D_{90}\right)$ was the same between the original and simulated plans. The relative dwell times were adjusted correspondingly although no re-optimization was performed.

For each original HDR treatment plan and each modified plan, the integral dose, highest dose delivered to $2 \mathrm{cc}\left(\mathrm{D}_{2 \mathrm{cc}}\right)$ of the bladder, bowel, sigmoid, rectum, and the ICRU rectum point dose were recorded. The ID was calculated by multiplying the source strength by the dwell time and represents the actual energy deposited in the tissue. In line with the ALARA principle, the ID to any uninvolved organ should be kept as low as reasonably achievable, as increased ID to normal structures near target volumes can result in toxicity or an increased risk for secondary malignancies. Thus, any change to integral dose is important to evaluate and was collected in this study. The dose delivered to the ICRU rectal point was used as a surrogate to evaluate vaginal toxicity [9]. The ICRU rectal point was placed during the retrospective modification of plans using the methods of the EMBRACE trial, and was reviewed by a board certified radiation oncologist [9].

The dose parameters captured were compared between the original and the modified plans using a paired two-sample $t$-test, with a $p$-value of $<0.05$ considered statistically significant.

\section{Results}

A total of 22 plans (11 original and 11 from reconstructed applicators' geometry) were analyzed. Table 2 lists the mean difference in dose between the original and the modified plans for all variables and the results of the paired two-sample $t$-test. The integral dose required to achieve a dose coverage of the HR-CTV that matched that of the initial dose prescribed was reduced by a mean of $6.1 \%$ in the $5 \mathrm{~mm}$ shifted plan $(p<0.001)$. The mean rectum $D_{2 c c}$ was $10.9 \%$ less in the modified plans when compared to the mean rectum $\mathrm{D}_{2 \mathrm{cc}}$ in the original treatment plans $(p=0.004)$. The mean $\mathrm{D}_{2 \mathrm{cc}}$ reductions for the remaining OARs and the ICRU rectum point ranged from $1.1 \%$ to $2.2 \%$. Among these values only the mean $\mathrm{D}_{2 \mathrm{cc}}$ reduction to the sigmoid of $1.8 \%$ was found to be significant $(p=0.04)$. The remainder were not found to be statistically significant. An illustration of the isodose modification observed on a given plan with and without the Smit sleeve in place is provided in Figure 3.

\section{Discussion}

In the present study, we quantified the impact of the distal shifts in the ovoids caused by the use of a Smit sleeve on the integral dose and the dose to OARs during endocavitary brachytherapy for LACC. The results of this dosimetric analysis confirmed that the Smit sleeve does have a dosimetric impact, which should be accounted for

Table 2. Mean difference in integral dose and OAR dose between the original and modified plan. Modification included a $5 \mathrm{~mm}$ proximal shift of ovoid source positions parallel to the tandem

\begin{tabular}{lcccc} 
Variable & Mean difference (cGy) & Average change (\%) & $T$-statistic (df) & $P$-value \\
\hline Integral dose & 265.9 & 6.10 & $9.13(10)$ & $<0.001$ \\
\hline$D_{2 c c}$ bladder & 6.2 & 1.10 & $1.03(10)$ & 0.33 \\
\hline$D_{2 c c}$ bowel & 5.4 & 1.60 & $1.71(8)$ & 0.13 \\
\hline$D_{2 c c}$ rectum & 50.5 & 10.90 & $3.65(10)$ & 0.004 \\
\hline$D_{2 c c}$ sigmoid & 8.3 & 1.80 & $2.31(10)$ & 0.04 \\
\hline ICRU rectal dose & 12.0 & 2.20 & $1.60(10)$ & 0.14
\end{tabular}



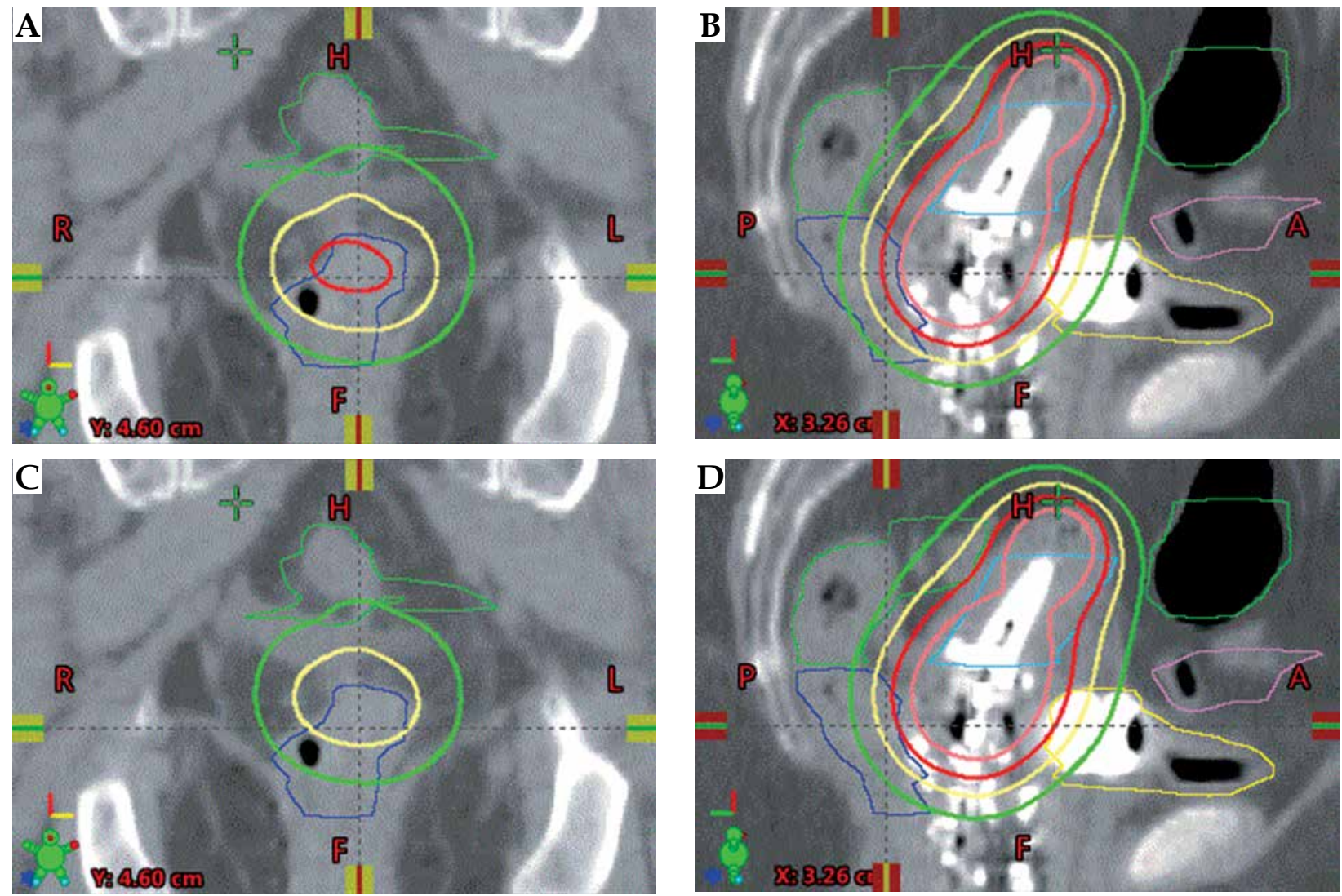

Fig. 3. A, B) Coronal and sagittal view of a plan with the sleeve present, no correction; C, D) coronal and sagittal view of a plan which mimics dosimetry without a Smit sleeve where ovoids were shifted $5 \mathrm{~mm}$ superiorly. The rectum, shown in blue, presents a notable reduction in volume receiving dose above the Rx dose (700 cGy red isodose line) in the plan mimicking a situation where the sleeve is not present

when a tandem and ovoids system is used for LACC endocavitary brachytherapy.

Numerous studies have evaluated the effects of source position shifts and obtained comparable results $[6,7,10]$. One study evaluating virtual tandem and ovoid shifts in the cranial and caudal setting found that a $3 \mathrm{~mm}$ shift of the applicator in the caudal direction resulted in a $10 \%$ change in the rectum $\mathrm{D}_{2 \mathrm{cc}}$, with similar lesser magnitude changes to the $\mathrm{D}_{2 \mathrm{cc}}$ to the bladder, sigmoid, and ICRU rectal dose [6]. Another study which evaluated shifts in the $x, y$, and $z$ axis and rotational shifts found that a $6 \mathrm{~mm}$ inferior shift increased the rectal dose by an average magnitude of $8.35 \%$ [10]. Furthermore, a study evaluating systematic applicator reconstruction uncertainties on dose volume histogram (DVH) parameters found that the rectal DVH shifted by approximately 3.5\% per mm shift in the longitudinal direction and that of the HR-CTV, bladder and sigmoid colon shifted $1 \%$ to $2 \%$ per $\mathrm{mm}$ shift in the longitudinal direction [7]. Although our study focused on the ovoid, rather than the entire applicator, shifts, similar outcomes were observed.

The magnitude of change in the rectum $\mathrm{D}_{2 \mathrm{cc}}$ was the most significant among the OARs. In cases where the Smit sleeve is utilized, caution should be taken to make sure the dose to the rectum is kept to a minimum [11]. Although the reduction in $\mathrm{D}_{2 \mathrm{cc}}$ was marginal for the remaining OARs, these additional doses to OARs may be of relevance for plans which are close to recommended dose constraints. Practitioners should be cognizant of these effects and evaluate the patient's anatomy prior to brachytherapy to determine whether distal displacement of the ovoids through the use of a Smit sleeve could impact the dose delivered to the OARs. In addition, they should consider using other techniques to reduce the dose to OARs such as speculum based vaginal packing [12], changes to bladder volume [13] or other techniques.

To conclude that the Smit sleeve is without benefit however would not be correct. Use of the Smit sleeve allows for easier insertion of the intrauterine tandem, decreasing the risk of uterine perforation, and provides an additional imaging marker to localize the cervix [11,14]. Given these benefits, providers may still elect to use the sleeve, either accepting the risk of a higher mean integral dose and OAR dose when used with a tandem and ovoids system. Moreover, if a Smit sleeve is necessary it might be better to consider using a tandem and ring applicator, since the Smit sleeve does alter the applicators' geometry in this case, as can be seen in Figure 1.

The development of a modified Smit sleeve with a reduced base height or a different anchoring system which would reduce the displacement of the ovoids might be a solution.

Finally, preset applicator model templates are sometimes used during catheter reconstruction, especially 
with the growth of 3D image based treatment planning $[15,16]$. Use of templates in which the ovoids shift, caused by the presence of the Smit sleeve, this not being taken into consideration, could lead to inaccurate assessment of the doses delivered to the HR-CTV. As previously reported, any impactful variation in catheter positioning on simulation should result in manual reconstruction of the catheters in the treatment plan $[7,17]$. Given the variation caused by Smit sleeves, catheters with Smit sleeves should be manually reconstructed.

Although this dosimetric analysis conveys the importance of accounting for the Smit sleeve when planning HDR brachytherapy with a tandem and ovoids system, it has several limitations. This analysis did not account for the lateral displacement of tissue caused by the cannula of the Smit sleeve. Additionally, contouring of the HR-CTV and other structures can differ between providers, which can alter the dosimetry [18]. This possible variation was not accounted for in this study. Future studies should attempt to evaluate the dosimetric effects of the Smit sleeve through phantom studies and prospective evaluation.

\section{Conclusions}

Use of a Smit Sleeve with a tandem and ovoids system increases the integral dose and might affect the dose delivered to the OARs, particularly the rectum. Use of this device should be carefully considered when planning HDR brachytherapy with a tandem and ovoids system. A tandem and ring applicator might be a better choice for these clinical situations.

\section{Disclosure}

The authors report no conflict of interest.

\section{References}

1. Banerjee R, Kamrava M. Brachytherapy in the treatment of cervical cancer: a review. Int J Womens Health 2014; 6: 555564.

2. Smit B. Design features of the indwelling intrauterine tube for high dose rate intracavitary therapy for carcinoma of the cervix and some hints on its optimal use. Br J Radiol 1993; 66: 1042-1043.

3. Smit B, du Toit J, Groenewald W. An indwelling intrauterine tube to facilitate intracavitary radiotherapy of carcinoma of the cervix. Br J Radiol 1989; 62: 68-69.

4. Tyrie L, Hoskin P. Intrauterine high dose rate afterloading brachytherapy: experience of fractionated therapy using a cervical sleeve technique. Clin Oncol 1996; 8: 376-379.

5. Awunor O, Berger D, Kirisits C. A multicenter study to quantify systematic variations and associated uncertainties in source positioning with commonly used HDR afterloaders and ring applicators for the treatment of cervical carcinomas. Med Phys 2015; 42: 4472-4483.

6. Schindel J, Zhang W, Bhatia SK et al. Dosimetric impacts of applicator displacements and applicator reconstruction-uncertainties on 3D image-guided brachytherapy for cervical cancer. J Contemp Brachytherapy 2013; 5: 250-257.

7. Tanderup K, Hellebust TP, Lang S et al. Consequences of random and systematic reconstruction uncertainties in 3D image based brachytherapy in cervical cancer. Radiother Oncol 2008; 89: 156-163.
8. Kirisits C, Rivard MJ, Baltas D et al. Review of clinical brachytherapy uncertainties: analysis guidelines of GEC-ESTRO and the AAPM. Radiother Oncol 2014; 110: 199-212.

9. Kirchheiner K, Potter R, Tanderup K et al. Health-related quality of life in locally advanced cervical cancer patients after definitive chemoradiation therapy including image guided adaptive brachytherapy: an analysis from the EMBRACE study. Int J Radiat Oncol Biol Phys 2016; 94: 1088-1098.

10. Yong JS, Ung N, Jamalludin Z et al. Dosimetric impact of applicator displacement during high dose rate (HDR) Cobalt-60 brachytherapy for cervical cancer: A planning study. Radiat Phys Chem 2016; 119: 264-271.

11. Nag S, Erickson B, Thomadsen B et al. The American Brachytherapy Society recommendations for high-dose-rate brachytherapy for carcinoma of the cervix. Int J Radiat Oncol Biol Phys 2000; 48: 201-211.

12. Sud S, Roth T, Jones E. Clinical analysis of speculum-based vaginal packing for high-dose-rate intracavitary tandem and ovoid brachytherapy in cervical cancer. J Contemp Brachytherapy 2018; 10: 32-39.

13. Sharma AD, Poddar J, Suryanarayan KU et al. Dosimetric analysis of the effects of the bladder volume on organs at risk (OAR) in high-dose-rate intracavitary brachytherapy in carcinoma cervix - an institutional study. J Contemp Brachytherapy 2018; 10: 26-31.

14. Jan YT, Chang CL, Tai HC et al. The air matters - sleeve air cavity as a marker guiding image-guided helical tomotherapy to target cervical cancer. J Contemp Brachytherapy 2016; 8: 82-87.

15. Richart J, Carmona-Meseguer V, García-Martínez T et al. Review of strategies for MRI based reconstruction of endocavitary and interstitial applicators in brachytherapy of cervical cancer. Rep Pract Oncol Radiother 2018; 23: 547-561.

16. Hellebust TP, Kirisits C, Berger D et al. Recommendations from Gynaecological (GYN) GEC-ESTRO Working Group: considerations and pitfalls in commissioning and applicator reconstruction in 3D image-based treatment planning of cervix cancer brachytherapy. Radiother Oncol 2010; 96: 153-160.

17. Humer I, Kirisits C, Berger D et al. Improved source path localisation in ring applicators and the clinical impact for gynecological brachytherapy. J Contemp Brachytherapy 2015; 7: 239-243.

18. Mashouf S, Safigholi H, Merino T et al. Sensitivity of clinically relevant dosimetric parameters to contouring uncertainty in postimplant dosimetry of low-dose-rate prostate permanent seed brachytherapy. Brachytherapy 2016; 15: 774-779. 